\section{PTH-123 IS AXIAL SPONDYLOARTHRITIS IN IBD DIFFERENT TO AXIAL SPONDYLOARTHRITIS WITHOUT IBD?}

1,3 Uma Selvarajah*, ${ }^{3}$ Jesus Miguens Blanco, 1,3 Shiva Radhakrishnan, ${ }^{1,3}$ Sam Powles, ${ }^{3} J u l i e$ McDonald, ${ }^{3}$ Alex Pechlivanis, ${ }^{3} J u l i a n$ Marchesi, ${ }^{1,3}$ Horace Williams, ${ }^{2,3}$ Andrew Keat, ${ }^{2}$ Pedro Machado, ${ }^{1,3}$ Timothy Orchard. 'Imperial College healthcare NHS trust, London, UK; ${ }^{2}$ London North West University Healthcare NHS Trust, Harrow, UK; ${ }^{3}$ Imperial College, London, UK

\subsection{6/gutjnl-2019-BSGAbstracts. 182}

Up to $40 \%$ of the patients with inflammatory bowel disease (IBD) have MRI evidence of axial spondyloarthritis compared to $1.5 \%$ in the non IBD population. Here we aim to distinguish axial spondyloarthritis associated with IBD (axSpA-IBD) from axSpA without IBD (axSpA-not IBD) and psoriatic axSpA (axSpA-PsA).

Method A collaborative group comprising of gastroenterologists and rheumatologists was formed between 2 healthcare trusts. Clinic lists and electronic operating systems were interrogated with appropriate ethical approval to identify patients with axSpA-IBD, axSpA-not IBD and axSpA-PsA. Patients were contacted prior to their clinic appointments and were sent stool containers. On the day of their clinic appointments, they were consulted for 15-30 minutes where their diseases were carefully phenotyped, drug history obtained, and informed consent taken. Stool and blood samples were collected and analysed for faecal calprotectin and acute inflammatory markers.

Results 62 patients were analysed for this study; 22 patients (10 Crohn's/12 UC) with axSpA-IBD, 24 with axSpA-not IBD and 16 patients with axSpA-PsA. Of the patients with IBD, 13 out of $22(59 \%)$ were diagnosed with IBD first. Median duration of IBD before the diagnosis of axSpA was 3.5 years. Remaining 9 patients who were diagnosed with axSpA first, the median duration of disease before the diagnosis IBD was 3.5 years as well.

Median age of onset for joint disease in axSpA-IBD is 30 years compared to 27 and 22 in axSpA-not IBD and axSpAPsA cohorts.

All three cohorts had gender predominance towards the male sex with $82 \%$ in axSpA-IBD, $86 \%$ in axSpA-not IBD and $94 \%$ in axSpA-PsA.

Association with HLA $\mathrm{B} * 27$ is stronger in the axSpA-not IBD group with $76 \%$ patients possessing the polymorphism compared to $50 \%$ in axSpA-IBD and $40 \%$ in axSpA-PsA. Median BASDAI (ankylosing spondylitis disease activity score) for axSpA-IBD, axSpA-not IBD and axSpA-PsA are 4.3, 3.95 and 3.2 respectively. Scores of above 4 indicate active disease.

Mean faecal calprotectin in axSpA-IBD is $233.7 \mathrm{ug} / \mathrm{g}$ compared to 99.1 and 28.2 in axSpA-not IBD and axSpA-PsA.

Pearson correlation coefficient between faecal calprotectin and BASDAI scores was 0.0451 .

Discussion In our cohort, we have shown that IBD related axial spondyloarthritis is different to axSpA-not IBD in terms of its weaker association with HLA $B * 27$ antigen, higher degree of gut inflammation and increased age of onset for joint disease. Mean faecal calprotectin in axSpA-not IBD was still significantly higher than axSpA-PsA.

Weaker association with HLA $B * 27$ antigen in axSpA-IBD points to the possibility of a different genetic loci or mode of aetiopathogenesis.
Contrary to what we had expected, male gender predominance, joint disease activity and functional disability are similar across both axSpA-IBD and axSpA-not IBD group. We have also shown that there is no correlation between gut inflammation and joint disease activity $(\mathrm{R}=0.0451)$ which again is consistent with previous findings that inflammatory axial disease is independent of gut inflammation.

\section{PTH-124 THE USE OF TACROLIMUS IN PATIENTS WITH ULCERATIVE COLITIS RESISTANT TO STANDARD MEDICAL THERAPY}

${ }^{1}$ Taimur Shafi*, ${ }^{2}$ Abdulla Bedir, 'Lucy Medcalf, ${ }^{1}$ Guy Chung-Faye, ${ }^{1}$ Benjamin Hope, ${ }^{1}$ Patrick Dubois, 'Bu Haye, 'Babu Vadamalayan, ${ }^{1}$ Alexandra J Kent. 'King's College Hospital, London, UK; ${ }^{2}$ Basildon and Thurrock University Hospital, Essex, UK

\subsection{6/gutjnl-2019-BSGAbstracts. 183}

Introduction BSG guidelines support the use of tacrolimus in selected adult or paediatric patients with ulcerative colitis (UC) resistant to standard therapy. Despite these guidelines, in our experience tacrolimus is used infrequently. We present the outcomes of $21 \mathrm{UC}$ patients commenced on tacrolimus at Kings College Hospital NHS Trust.

Methods The King's College Hospital electronic patient records database was searched, including all records from January 2011 to October 2018. The search was performed using the terms, 'ulcerative colitis' and 'tacrolimus.' All records were interrogated to collect retrospective data.

Results Our search yielded a total of 275 patients. 191 were excluded as they were on tacrolimus for an indication other than UC. A further 59 were discounted as tacrolimus was never commenced. 4 were removed where tacrolimus topical ointment was used.

21 UC patients (16 adult, 5 paediatric) treated with tacrolimus were identified. Mean age was 29 years (range 9 - 62), M:F 13:8, 13 Caucasian, 4 Afro-Caribbean, 2 Asian and 2 unspecified. $81 \%$ had pancolitis, 3 left-sided disease and 1 proctitis.

All 21 patients had previously been treated with 5 -aminosalicylates (5-ASAs) and steroids. In addition to the 5-ASAs and steroids: 12 patients were treated with both azathioprine (Aza) and a biologic; 4 patients only received Aza; 2 only a biologic; 1 only mycophenolate mofetil; 1 only mercaptopurine and 1 had no information.

Mean duration on tacrolimus was 11 months (median 5; range, $0-66$ ). 2 patients were primary non-responders and 9 terminated therapy due to adverse drug effects such as tremor, nausea and renal toxicity. $10(48 \%)$ patients had a sustained clinical response remaining on tacrolimus for a mean of 21 months. 1 remains on tacrolimus currently, but 9 patients have had disease recurrence, with 6 patients requiring a colectomy, at an average of 16 months after starting tacrolimus.

Faecal calprotectin (FCP) was used as an objective measure of disease activity. In patients on tacrolimus for a sustained period $(n=10)$, there was a significant reduction in FCP. Mean FCP levels pre- and during treatment were 2574 and 381 respectively (mean difference 2197, CI 3237 to -1157 , $\mathrm{p} 0.001)$ [figure 1]. 


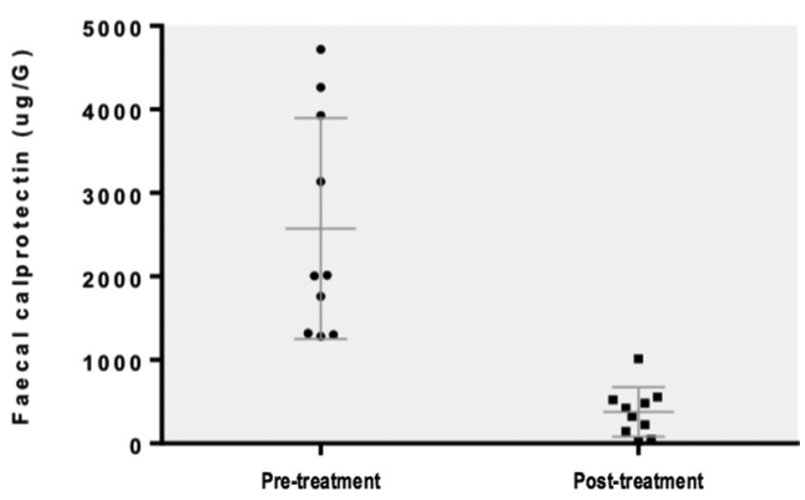

Abstract PTH-124 Figure 1 Comparison of patient faecal calprotectin levels before

Conclusion If tolerated, tacrolimus can be an effective treatment option for patients with ulcerative colitis. This response was maintained for an average of 21 months, with a significant drop in faecal calprotectin levels.

\section{PTH-125 THE CLINICAL UTILITY AND DIAGNOSTIC ACCURACY OF FAECAL CALPROTECTIN FOR IBD IN PAEDIATRIC PATIENTS}

Anet Soubieres, Benjamin Shandro*, Jai Mathur, Frances Boa, Thankam Paul, Andrew Poullis. St George's University Hospitals NHS Foundation Trust, London, UK

\subsection{6/gutjnl-2019-BSGAbstracts. 184}

Introduction Faecal calprotectin (FCP) has an established place in the adult diagnostic pathway. Its role in the paediatric population, where triage for colonoscopy is vital, is less well studied. There is increasing awareness that the normal range for FCP and the prevalence of IBD vary with age. We aimed to determine the most effective use of FCP in paediatric patients presenting with GI symptoms.

Methods We conducted a retrospective analysis of FCP results for patients aged $\leq 18$ years presenting to paediatric gastroenterology at a London teaching hospital from 2013 to 2014. Demographic and clinical information, including final diagnosis of IBD, was extracted from the Electronic Patient Record. In patients with multiple FCP results, the earliest was used. Abnormal FCP was defined as $\geq 50 \mu \mathrm{g} / \mathrm{g}$. Contingency tables for FCP and IBD were generated for the total cohort and grouped by age $<10$ years and $\geq 10$ years. Sensitivity, specificity, positive predictive values (PPVs), negative predictive values (NPVs) and pre- and post-test probabilities were calculated.
The analysis was repeated in patients aged $<10$ years using an FCP threshold $\geq 160 \mu \mathrm{g} / \mathrm{g}$, which has been adopted locally as the cut-off in this group. Complete case analyses were used where data were missing. Stata version 13.1 was used for all statistical analyses.

Results 356 FCP samples were sent from 328 patients. 49.9\% were male, and median age was 10.9 years (range 0.1-18.7). 134 patients (41\%) had an abnormal FCP. 90 patients $(27.4 \%)$ were diagnosed with IBD. The median FCP for patients with IBD was $408.5 \mu \mathrm{g} / \mathrm{g}$ vs. $18 \mu \mathrm{g} / \mathrm{g}$ for those without IBD. Using an FCP threshold of $\geq 50 \mu \mathrm{g} / \mathrm{g}$ for IBD vs. nonIBD the overall sensitivity was $76.7 \%$, specificity $72.4 \%$, PPV $52.6 \%$ and NPV $88.6 \%$. In patients $<10$ years old, the sensitivity was $100 \%$, specificity $70.2 \%$, PPV $21.7 \%$ and NPV $100 \%$. The pre-test probability was $7.6 \%$ (low) and post-test probability $21.6 \%$. In patients $\geq 10$ years old, the sensitivity was $73.8 \%$, specificity $75 \%$, PPV $69.4 \%$ and NPV $78.8 \%$. The pre-test probability was $43.5 \%$ (intermediate) and posttest probability $69.3 \%$. Increasing the FCP threshold to $\geq 160 \mu \mathrm{g} / \mathrm{g}$ in patients $<10$ years old improved the specificity to $85.1 \%$, but at the expense of sensitivity, which decreased to $80 \%$. The PPV was $30.8 \%$, NPV $98.1 \%$ and post-test probability $30.6 \%$. Colonoscopy was carried out in 133 patients (42\%), median age 12.5 years (range 0.2-18.5), of which 49 (36.8\%) had a normal FCP.

Conclusions FCP is highly accurate at excluding IBD in paediatric patients with GI symptoms and should guide the need for colonoscopy. In low prevalence populations, such as those aged $<10$ years, a positive result should be interpreted with caution.

\section{PTH-126 AUDIT OF BIOLOGICAL THERAPY FOR INFLAMMATORY BOWEL DISEASE: RESULTS FROM THE UK IBD REGISTRY}

${ }^{1}$ Mustafa Shawihdi ${ }^{*},{ }^{2,3}$ Fraser Cummings, ${ }^{2,4}$ Stuart Bloom, ${ }^{1}$ Keith Bodger. ${ }^{1}$ University Of Liverpool, Liverpool, UK; ${ }^{2}$ UK IBD Registry, Epsom, UK; ${ }^{3}$ University Hospitals Southampton, Southampton, UK; ${ }^{4}$ University College Hospital London, London, UK

\subsection{6/gutjnl-2019-BSGAbstracts. 185}

Introduction Ensuring the safe, appropriate and effective use of costly biological agents presents a significant challenge for healthcare systems. Although no longer funded as a national audit programme, NHS England has identified audit of biologics for IBD as a priority area for QI activity for hospitals (Quality Accounts List). The UK IBD Registry provides a system for collecting, submitting and reporting data to support participation in biologics audit.

Methods Participating centres submit quarterly extracts of standardised data collected via a range of software solutions,

\section{Abstract PTH-126 Table 1}

\begin{tabular}{|c|c|c|c|}
\hline Key Performance Indicator, mean $\%$ (\% sites below mean) & $\begin{array}{c}\text { All Sites } \\
(n=65)\end{array}$ & $\begin{array}{c}0-19 \text { cases } \\
(n=22)\end{array}$ & $\begin{array}{c}\geq 20 \text { cases } \\
\quad(n=43)\end{array}$ \\
\hline 1 Infection screening before starting drug (naïve only) & $69.7(31 \%)$ & 75.4 & 69.5 \\
\hline 2 Assessment of disease activity (pre-treatment) & $35.7(38 \%)$ & 39.5 & 35.6 \\
\hline 3 Registry consent recorded & 39.1 (41\%) & 32.9 & 39.4 \\
\hline 4 Post-induction review recorded & $38.4(45 \%)$ & 36.9 & 38.5 \\
\hline 5 Assessment of disease activity (post-induction review) & $38.3(25 \%)$ & 35.3 & 38.4 \\
\hline 6 Twelve month review recorded & $34.5(32 \%)$ & 15.4 & 35.3 \\
\hline 7 Assessment of disease activity (twelve month review) & $40.0(20 \%)$ & 33.3 & 40.2 \\
\hline
\end{tabular}

